\title{
Finite Element Analysis of Masticatory Stress on Neoformed Bone Tissue After Distraction Osteogenesis and Low-Level Laser Therapy: A Pilot Study
}

\author{
Angelo Luiz Freddo, $\mathrm{PhD}$, Eliete Biasotto Hauser, $\mathrm{PhD}{ }^{2}$ Victor Velho de Castro, ${ }^{3}$ \\ Pedro Yoshito Noritomi, PhD, ${ }^{4}$ Alexandre Simões de Almeida, ${ }^{4}$ and Marília Gerhardt de Oliveira, $\mathrm{PhD}^{5}$
}

\begin{abstract}
Objective: This study aimed to understand the action of masticatory forces on an implant virtually introduced into the sheep mandible after distraction osteogenesis and low-level laser therapy (LLLT) by using finite element analysis. Background data: Distraction osteogenesis as an alternative for bone reconstruction that may be used in the treatment of deformities. Methods: Four ewes underwent distraction osteogenis to elongate the left mandibular body by $15 \mathrm{~m}$, and three of them underwent LLLT with the purpose of improving bone properties. After death, animals were scanned by computed tomography and their mandibles were tridimensionally reconstructed by computer programs. The physical properties related to hardness and modulus of elasticity of each animal were obtained from the dissected mandibles, and data were transferred to Femap software for finite element analysis. Results: Animals exposed and not exposed to LLLT irradiation showed remarkably similar values for superficial hardness and modulus of elasticity, without statistically significant difference $(p>0.05)$, between the values observed for the cortical bone and the cancellous bone among the groups. The neoformed mandible, after a brief period for bone healing, was able to promote stability for implant placement and proper distribution of masticatory forces. Conclusions: An implant introduced virtually into the site of bone neoformation did not suffer any micromotions relevant to osteointegration. Furthermore, finite element analysis showed that the neoformed portion of the mandible was able to absorb and distribute masticatory forces throughout its structure, even after a brief period for bone maturation.
\end{abstract}

\section{Introduction}

D ISTRACTION OSTEOGENESIS (DO) IS A PROMISING ALTERNATIVE for facial reconstructive surgery, with applications in cases of congenital malformations, trauma, post-oncologic surgery reconstruction, and prosthetic rehabilitation of oral and maxillofacial defects with osseointegrated implants. The use of DO in bone reconstruction in preparation for later rehabilitation with dental implants is becoming increasingly popular. One inconvenience of this approach is the time required for bone maturation, during which patients cannot yet receive osseointegrated dental implants.

DO uses external or internal distraction devices to enable bone growth at deformed sites by stimulating the body's own mechanisms for bone and soft tissue repair. ${ }^{1,2}$ The prolonged bone maturation periods of DO and the long-term stability of its outcomes have yet to be well documented; instability and recurrence have been reported. ${ }^{3,4}$ Therefore, strategies that can speed up bone maturation and improve the physical properties of the elongated bone site are an area of research interest.

Low-level laser therapy (LLLT) is being studied as a potential means of biomodulating inflammation and bone repair, in view of its photochemical and photobiological properties, and of the hypothesis that laser therapy might speed bone repair, decrease discomfort and edema, and improve tissue healing after surgery. ${ }^{5-7}$ In this regard, previous studies have assessed the quality of bone healing ${ }^{8,9}$ after DO and LLLT. ${ }^{10-12}$

\footnotetext{
${ }^{1}$ School of Dentistry, Universidade Federal do Rio Grande do Sul, Porto Alegre, Brazil.

${ }^{2}$ School of Mathematics, Pontifícia Universidade Católica do Rio Grande do Sul (PUCRS), Porto Alegre, Brazil.

${ }^{3}$ School of Mechanical Engineering, Pontifícia Universidade Católica do Rio Grande do Sul (PUCRS), Porto Alegre, Brazil.

${ }^{4}$ Centro de Tecnologia da Informação Renato Archer - CTI, Campinas, Brazil.

${ }^{5}$ Research Productivity Fellow, CNPq, Porto Alegre, RS, Brazil.
} 
Finite element analysis (FEA - for a review of its mathematical foundations, see ${ }^{13,14}$ ) provides a means of solving problems that involve force and stress and their respective interactions. ${ }^{15,16}$ As in vivo stress analysis is essentially infeasible, computational modeling is an increasingly frequent strategy for analysis of the distribution of these forces at the bone-implant interface. Determination of the elastic properties of bone and of the stresses occurring within these structures is extremely important, as a wide range of scientific studies have provided evidence of failures in the bone regeneration process.

The aim of this study is to present the findings of a finite element simulation of dental implant placement in an area of neoformed bone, after DO with a brief consolidation period and LLLT, in an experimental animal model, and to conduct an analysis of stress distribution and implant displacement under the action of vertical, horizontal, and oblique occlusal loads in this setting.

The specific aims are to compare bone physical properties (modulus of elasticity) of animals with and without exposure to LLLT. Moreover, this study seeks to observe the behavior of the neoformed bone/implant structure with different periods of bone consolidation during masticatory stress.

The null hypothesis of this research considers that LLLT has no biological effect on speeding the maturation process of the neoformed bone; therefore, this bone may not be able to withstand and distribute the masticatory force of the implant introduced by virtual simulation.

\section{Materials and Methods}

This project was approved by the relevant Research Ethics Committee and Animal Experimentation Ethics Committee with judgment no. CEUA 08/00040.

Four ewes (age 2 years, weight $40-45 \mathrm{~kg}$ ), designated $E 1$, $E 2, E 3$, and $E 4$, were selected for experimentation. General anesthesia was used and pre- and postoperative medication was administered by staff veterinarians as per hospital protocols. After induction of anesthesia, animals were placed in right lateral decubitus position, and the left submandibular region was exposed. Corticotomies were performed with a reciprocating saw through the medial and lateral aspects of the mandible, toward the retromolar triangle, and joined at the basilar edge and alveolar border, near the angle of the mandible. The distraction device was positioned and installed with four screws, and the surgical wound was closed with simple interrupted sutures (4/0 monofilament nylon), which were removed on postoperative day 7 .

Three animals were exposed to LLLT irradiation with the purpose of improving bone physical properties (nanohardness and modulus of elasticity), ${ }^{9}$ given the brief period for bone consolidation after DO. LLLT was performed using an active laser medium [aluminium gallium arsenide (AlGaAs), wavelength $830 \mathrm{~nm}, 35 \mathrm{~mW}$ ]. Ewes 1 and 2 were irradiated immediately after closure of the surgical wound and $48 \mathrm{~h}$ thereafter for a total of eight sessions. Spot laser was applied to three points at an energy density of $5 \mathrm{~J} / \mathrm{cm}^{2}$, in continuous mode $(35 \mathrm{~mW})$, for a total dose of $15 \mathrm{~J} / \mathrm{cm}^{2}$. Total application time was 2.23 min. Ewe 3 was subjected to a similar irradiation protocol, but the first session took place on the 1 st day of bone maturation. Ewe 4 was not irradiated.
The materials and protocols used for DO and a full description of the methods of this study have been published elsewhere. $^{17}$

\section{DO protocol}

The DO protocol was as follows:

1. Latency period: 5 days

2. Activation period: 15 days (device activation at a rate of $1 \mathrm{~mm} /$ day).

3. Bone consolidation period: 30,20 , and 13 days.

The distractor remained inactive for 30 days in ewe 1, for 20 days in ewe 2, and for 13 days in ewes 3 and 4 for bone consolidation to occur. Following the bone maturation period assigned to each group, the distraction device was removed with the animal under local anesthesia. Sixty days after surgery, the animals were euthanized by deep anesthesia. Mandibles were skeletonized, fixed in glutaraldehyde, scanned by computed tomography $(0.5 \mathrm{~mm}$ thick axial slices), and sent for nanohardness testing at the Materials and Nanosciences Laboratory.

\section{Mechanical and morphological assessment of neoformed bone}

The mechanical properties of neoformed bone were assessed by measurement of nanohardness and modulus of elasticity, using a Berkovich tip and a dynamic nanoindenter with a displacement resolution of $2 \mathrm{~nm}$ and a load resolution of $20 \mathrm{~N}$. Load was applied linearly at a standardized rate of $50 \mathrm{mN} / 250 \mathrm{~nm}$ over the external and internal cortical surfaces, from $0.5 \mathrm{~mm}$ before to $0.5 \mathrm{~mm}$ beyond the neoformed region, enabling continuous measurement of elastic properties from the bone area subjected to DO all the way to normal mature mandibular bone. The data obtained from nanohardness testing, such as universal hardness and modulus of elasticity, provided bone properties for calculation of the finite element model.

\section{Construction of finite elements}

The technical approach used in this study for finite element analysis involves generation of three-dimensional (3D) biomodels from two-dimensional (2D) DICOM files of computed tomography scans, using the Invesalius ${ }^{\mathrm{TM}} 2.1$ software package.*1 The 2D DICOM file sequences were imported into Invesalius and reconstructed to generate $3 \mathrm{D}$ models consisting of point clouds, which were then discretized by triangulation and used to generate STL files.

STL files were imported into Rhinoceros 3D (Fig. 1A) for mandibular surface modeling. A titanium implant connected to a porcelain-fused-to-metal crown, designed to meet the parameters of implants commonly used in dental practice, was also modeled and implanted virtually into the neoformed bone, as if it were completely osseointegrated (Fig. 1B).

The finite element chosen for modeling was a 10 node or quadratic tetrahedron, which, geometrically, consists of a triangular pyramid with one node at each vertex and one node at the center of each edge. Before calculation, the geometry of each

\footnotetext{
"Downloaded from the Brazilian Public Software Portal (http:// www.softwarepublico.gov.br/).
} 
FIG. 1. (A) Base geometry, .STL file. (B) Mandible model. MB, medullary (cancellous) bone; $\mathrm{CB}$, cortical bone; I, implant and crown; $\mathrm{OD}$, distraction line.
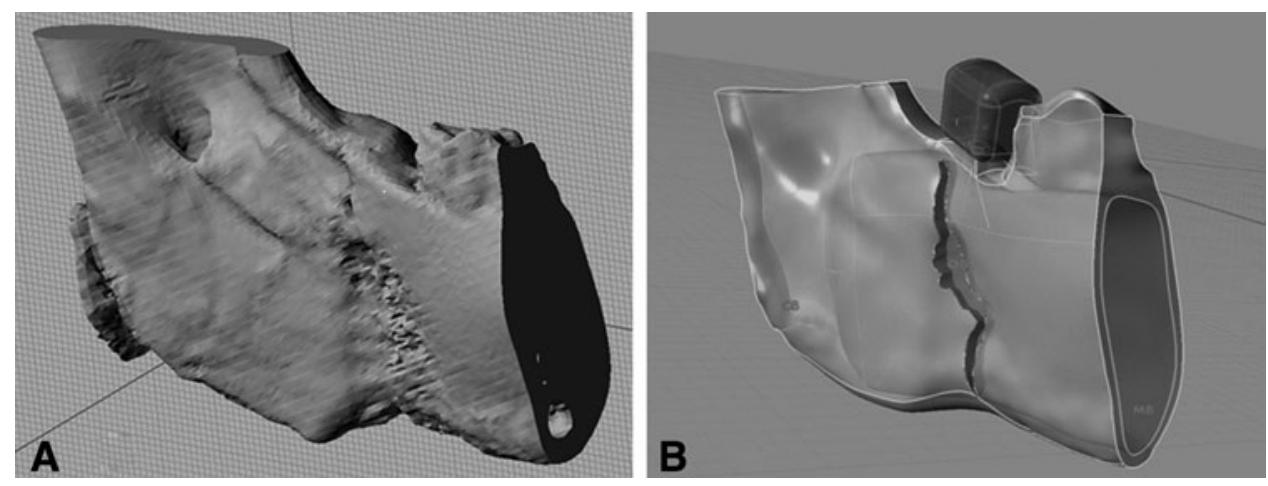

mandibular model was checked thoroughly to assess whether any adjustments through CAD remodeling were required.

CAD models were then exported to the Femap software environment, where files were preprocessed; model quality was checked to ensure efficient mesh generation; and areas of restricted range of motion, masticatory loading regimes, and the properties of all materials and biological tissues were determined.

Assessment of stresses in an asymmetrical model requires information known as "input data," so that the software can process and calculate stress by simulation of the action of masticatory force on the implant and of the force of the implant on bone tissue. The input data used in this study, including the properties of all elements used in the computational model, were based on the literature ${ }^{18-20}$ and on actual laboratory data obtained from measurements of sheep subjected to DO, and are listed in Table 1. According to Nagasao et al., ${ }^{21}$ the masticatory force generated in the molar region ranges from 75 to $300 \mathrm{~N}$. The horizontal $(30 \mathrm{~N})$, vertical $(105 \mathrm{~N})$ and oblique $(210 \mathrm{~N})$ masticatory loads used by Veziroglu and Yilmaz ${ }^{20}$ in their study were the same that were applied on the implant crown in the simulation performed in

Table 1. Material Properties

\begin{tabular}{lcc}
\hline & $\begin{array}{c}\text { Modulus } \\
\text { of elasticity } \\
\text { Material }\end{array}$ & $\begin{array}{c}\text { Poisson's } \\
\text { ratio }\end{array}$ \\
\hline Titanium & $110^{\mathrm{a}}$ & $0.33^{\mathrm{a}}$ \\
Ceramic (artificial tooth) & $170^{\mathrm{a}}$ & $0.22^{\mathrm{a}}$ \\
Nickel-chromium alloy & $188^{\mathrm{a}}$ & $0.28^{\mathrm{a}}$ \\
Normal cortical bone (S1) - 2mm & 18.42 & $0.3^{\mathrm{a}}$ \\
Normal cortical bone (S2) - 2mm & 18.10 & $0.3^{\mathrm{a}}$ \\
Normal cortical bone (S3) - 2mm & 18.78 & $0.3^{\mathrm{a}}$ \\
Normal cortical bone (S4) - 2mm & 18.10 & $0.3^{\mathrm{a}}$ \\
Cancellous bone (S1) & 1.84 & $0.34^{\mathrm{a}}$ \\
Cancellous bone (S2) & 1.81 & $0.34^{\mathrm{a}}$ \\
Cancellous bone (S3) & 1.87 & $0.34^{\mathrm{a}}$ \\
Cancellous bone (S4) & 1.81 & $0.34^{\mathrm{a}}$ \\
Neoformed bone (cortical) (S1) & 11.06 & $0.33^{\mathrm{a}}$ \\
Neoformed bone (cortical) (S2) & 10.92 & $0.33^{\mathrm{a}}$ \\
Neoformed bone (cortical) (S3) & 12.36 & $0.33^{\mathrm{a}}$ \\
Neoformed bone (cortical) (S4) & 12.02 & $0.33^{\mathrm{a}}$ \\
Neoformed bone (cancellous) (S1) & 1.10 & $0.33^{\mathrm{a}}$ \\
Neoformed bone (cancellous) (S2) & 1.09 & $0.33^{\mathrm{a}}$ \\
Neoformed bone (cancellous) (S3) & 1.23 & $0.33^{\mathrm{a}}$ \\
Neoformed bone (cancellous) (S4) & 1.20 & $0.33^{\mathrm{a}}$ \\
\hline
\end{tabular}

${ }^{a}$ Literature data.

S1, sample 1; S2, sample 2; S3, sample 3; S4, sample 4. this study. Loads were exerted separately in these three directions, on 10 points over the surface of the implant crown. The thickness of mandibular cortical bone was considered to be $2 \mathrm{~mm}$. Sites of restricted mandibular motion were placed in the anterior region of the mandible, to simulate locking of the masseter muscle, and in the mandibular ramus, to simulate the lateral pterygoid muscle.

After loading of models, the NEi Nastran software suite was used for calculation of finite elements. This calculation involves subdivision of the model into a mesh of simpler geometric elements, enabling calculation of the balance of forces acting upon each of these elements. Nastran provides the results of these equations for each region of the mesh, but cannot plot a graphic color map of these results on the specimen model; this requires postprocessing, which was performed with Femap software. The color maps yielded by this process are shown in Figs. 2 and 3.

\section{Data analysis: biomechanical simulations}

Biomechanical simulations are meant to simulate the stresses involved in masticatory loads and exerted on the implant, along their various directions, and to observe the behavior of neoformed mandibular bone. Implant displacement (micromotions), von Mises stress, maximum principal stress, and maximum principal stress in bone were analyzed.

\section{Results}

Animals that were exposed to LLLT irradiation, both in the activation period and in the consolidation period, and animals that were not exposed to LLLT irradiation showed remarkably similar values for superficial hardness and modulus of elasticity, without statistically significant difference $(p>0.05)$, between the values observed for One important measure that can help bring results closer to reality is to use the greatest possible number of elements. The number of elements used in this study was quite high (Table 2) and mesh creation sought to strike a balance between available computational resources and consistency of results, suggesting that results are reasonable estimates of reality. In this research, the neoformed mandibular tissue after a brief period of bone consolidation was able to hold an implant during masticatory function without compromising the bone/implant structure, as is shown in Figs. 2 and 3.

The maximum values obtained in each analysis, as well as their location on the implant/mandible system, are shown in Table 3. 

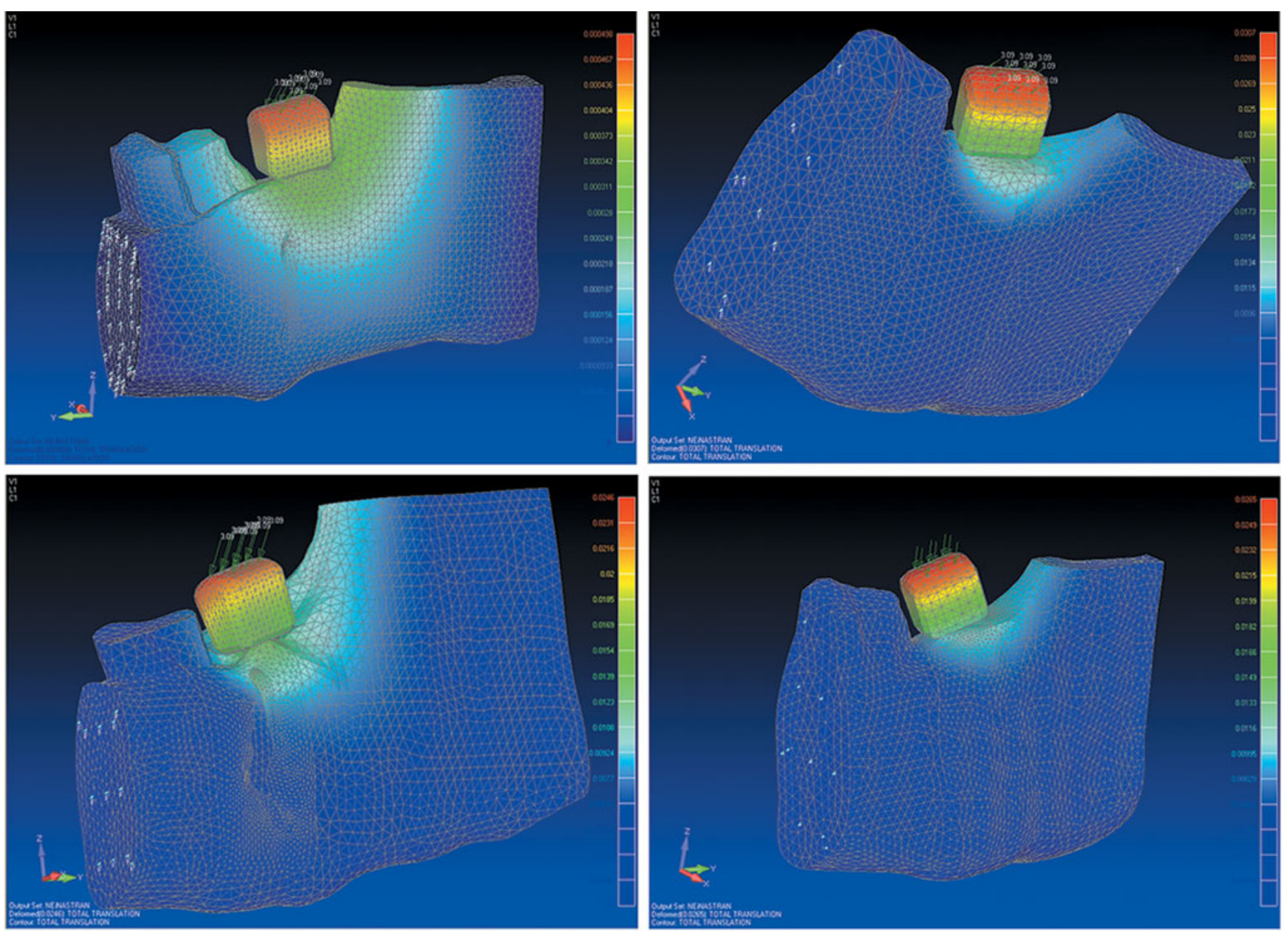

FIG. 2. Distribution of forces across the mandibular structure under an oblique load.

\section{Discussion}

The mandible actually comprises two bony structures with distinct features: cortical bone and cancellous bone. Furthermore, in the setting of this study, mandibles also had neoformed cortical and cancellous bone, with distinct physical properties. This complex anatomy of the mandibular bone and the presence of countless parts and components in the present biomodels mandated the use of a large quantity of elements and nodes, making construction of each biomodel challenging, and requiring a vast number of computer hours.

In an attempt to obtain actual data, all of these structures were modeled separately and considered separately for construction of the finite element mesh. Hardness and modulus of elasticity were measured under laboratory conditions (nanohardness testing) rather than drawn from the literature, increasing the reliability of the experiment. To simulate real-life masticatory action, areas of restricted range of motion were simulated in the anterior region of the mandible, to emulate the action of the masseter muscle, and in the region of the temporal muscle-as these two muscles account for most of the load exerted on the mandible during the mastication process-as well as the main muscles responsible for connecting the mandible to the cranium.
However, the complexity of mandibular motion, which involves different muscle actions and insertions that can be easily reproduced by virtual simulation, represents the greatest limitation of this type of experiment. Additionally, there is the limitation of generating extremely complex artificial structures; therefore, the implant-mandible system should be simplified.

According to Duyck et al., ${ }^{22}$ the longitudinal predictability and success of treatment are highly influenced by the biomechanical environment to which the implant is exposed. The concentration of loads (forces and deformations) applied to bone may cause a buildup of microdamage and induce bone resorption. For measurement of these stresses and displacements in the present simulation, the masticatory forces generated at a molar were used as the load for simulation, as described in the literature by many authors, such as Nagasao et al. ${ }^{21}$ and Veziroglu and Yilmaz. ${ }^{20}$

To Mehra and Figueroa, ${ }^{1}$ DO is a surgical technique that takes advantage of the body's own repair mechanisms for reconstruction of soft tissue and bone. In this study, mandibles were elongated by $15 \mathrm{~mm}$, and neoformed bone was subjected to nanohardness testing after only 30, 20, and 13 days of consolidation. According to Ni et al., ${ }^{23}$ nanohardness is an indicator of the resistance of a material to penetration and plastic deformation. Nanohardness testing 

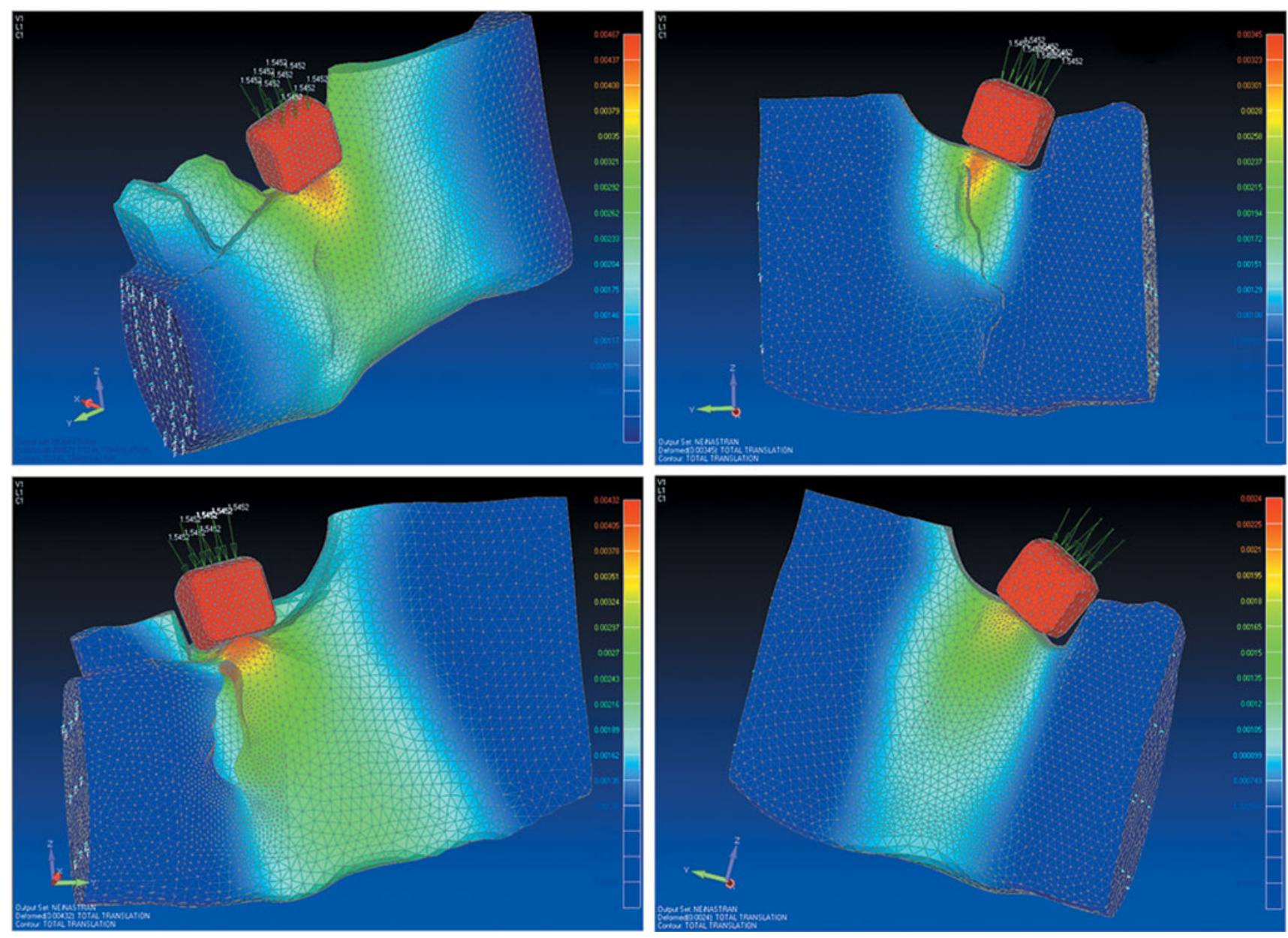

FIG. 3. Concentration of stress on the implant crown under a vertical load.

reveals mechanical properties such as apparent modulus of elasticity, deformations, and residual differences in analyzed areas. In this sample, the hardness and elasticity of neoformed bone were similar in all animals, showing that LLLT was not able to improve the physical properties of the tested samples, as shown in Table 1.

It bears noting that the long-term stability of DO outcomes is not assured; there have been reports of recurrence. This highlights the fact that, although neoformed bone is ready for loading after a minimum maturation period of 1330 days, there is no way of confirming whether regression of the elongation of said bone (recurrence) may occur as a result of an insufficient consolidation period. In this regard, many studies have sought ways of speeding the bone maturation process and improving the physical properties of the elongated bone site, whether by laser therapy, magnetic fields, or BMPs. ${ }^{10-12,24}$

A qualitative analysis of the finite element model (FEM) shows that no loads generated critical stress, and that all masticatory forces were well tolerated and distributed by and across the dental implant and the neoformed bone (Fig. 2).

Corroborating these results, simulated implant placement in the region of a molar overlying neoformed bone tissue after a brief consolidation period and LLLT showed the ability to disperse all masticatory forces without critical stress at any point in the structure of the mandible. In all studied animals and in all directions, the sole point at which stresses concentrated was the crown of the implant, as expected, as this was the point on which force was applied. The temporomandibular joints and masticatory muscles

Table 2. Number of Elements and Nodes Used for Finite Element Mesh Generation

\begin{tabular}{lllrr}
\hline Sheep & Duration of distraction & Application of laser therapy & No. of elements & No. of nodes \\
\hline 1 & 50 days & Latency/activation & 114,638 & 182,069 \\
2 & 40 days & Latency/activation & 78,572 & 127,672 \\
3 & 33 days & Bone consolidation & 101,933 & 163,638 \\
4 & 33 days & - (No therapy) & 86,700 & 139,600 \\
\hline
\end{tabular}


Table 3. Stresses and Displacements According to the Finite Element Method

\begin{tabular}{|c|c|c|c|c|}
\hline Animal, stress & $\begin{array}{c}\text { Max. von } \\
\text { Mises stress }(M P a)\end{array}$ & $\begin{array}{l}\text { Max. principal } \\
\text { stress }(M P a)\end{array}$ & $\begin{array}{c}\text { Max. implant } \\
\text { displacement }(\mu m)\end{array}$ & $\begin{array}{l}\text { Max. principal stress } \\
\text { in bone }(M P a)\end{array}$ \\
\hline E1, Horizontal & 14.65 & 38.22 & 6.24 & 3 \\
\hline Location & Implant platform & Base of crown & Top of crown & Sites of restricted motion, implant site \\
\hline E1, Oblique & 1 & 2.618 & 0.49 & 0.253 \\
\hline Location & Implant platform & Base of crown & Top of crown & $\begin{array}{l}\text { Sites of restricted motion, neoformed } \\
\text { bone }\end{array}$ \\
\hline E1, Vertical & 9.858 & 4.456 & 4.67 & 4.456 \\
\hline Location & Implant platform & $\begin{array}{l}\text { Sites of restricted } \\
\text { motion }\end{array}$ & Whole crown & Sites of restricted motion \\
\hline E2, Horizontal & 31.78 & 52.96 & 5.96 & 3.75 \\
\hline Location & Implant platform & Base of crown & Top of crown & Implant site \\
\hline E2, Oblique & 162.35 & 175.56 & 30.7 & 7.26 \\
\hline Location & Implant platform & Base of crown & Top of crown & $\begin{array}{l}\text { Sites of restricted motion, } \\
\text { neoformed bone, implant site }\end{array}$ \\
\hline E2, Vertical & 12.7 & 4.595 & 3.45 & 4.595 \\
\hline Location & Implant platform & Alveolar ridge & Whole crown & Alveolar ridge \\
\hline E3, Horizontal & 59.02 & 24.58 & 22.8 & 20.26 \\
\hline Location & Implant platform & Base of crown & Top of crown & Sites of restricted motion, implant site \\
\hline E3, Oblique & 65.4 & 128.8 & 24.6 & 13.65 \\
\hline Location & Implant platform & Base of crown & Top of crown & Sites of restricted motion \\
\hline E3, Vertical & 12.5 & 3.75 & 4.32 & 3.75 \\
\hline Location & Implant platform & $\begin{array}{l}\text { Superior aspect } \\
\text { of neoformed bone }\end{array}$ & Whole crown & Alveolar ridge, neoformed bone \\
\hline E4, Horizontal & 21 & 7.927 & 5.27 & 3 \\
\hline Location & Implant platform & Base of crown & Top of crown & Implant site \\
\hline E4, Oblique & 104.6 & 129.6 & 26.5 & 12.8 \\
\hline Location & Implant platform & Base of crown & Top of crown & Implant site \\
\hline E4, Vertical & 9 & 3 & 2.4 & 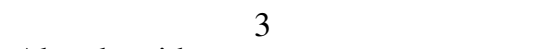 \\
\hline Location & Implant platform & Alveolar ridge & Whole crown & Alveolar ridge \\
\hline
\end{tabular}

promote complex movements and expose the teeth to loads of varying intensity and direction. The FEM showed that, in all groups, vertical loads were most relevant to the prosthesisimplant-bone complex, but all stresses were correctly dissipated from the crown of the implant to neoformed bone tissue and from there to mature bone (Fig. 3).

Von Mises stress analysis was only conducted on the implant, as it was the only part of the simulation whose material exhibits yield. As shown in Table 1, the greatest von Mises stresses were measured at the implant platform. These stresses were greatest under loads involving a horizontal component (oblique and horizontal loads), as shear stress (which is most relevant to the calculation of von Mises stress) arises from these forces.

Analysis of maximum principal stress included all anatomical specimens except the implant, because of the ductility of titanium. Maximum principal stress analysis is used for comparison of stresses between brittle and hard materials, such as bone and ceramic. Under horizontal and oblique loads, maximum stress was detected at the ceramic base of the crown. Maximum stress was not detected under vertical loads, which were always applied to bone tissue, but remained within the noncritical range throughout.

Deflection, or displacement, concerns the translational displacement occurring in each element of the structure. It is an important parameter when failure criteria are associated with displacement or when the conditions for application are restricted by displacement. In osseointegrated implants, for example, micromotions $>100 \mu \mathrm{m}$ may lead to formation of fibrous connective tissue instead of bone regeneration and osseointegration. $^{25}$ As Table 3 shows, no implant displacement $>30.7 \mu \mathrm{m}$ was detected in any of the animals. This means that critical displacement did not occur in any animals under any loads, which highlights the quality of neoformed bone.

Maximum principal stress was also measured solely in bone (both neoformed and mature), that is, not taking the ceramic crown or titanium implant into account. Analysis showed that stress buildup occurred mainly at the sites of restricted motion, at the implant site (socket), at the alveolar ridge, and in neoformed bone.

Table 3 shows the location of each point of maximum stress, but absolute (maximum and minimum) stress values should always be analyzed cautiously. The geometric factor associated with the anatomical landmarks of each mandible is a major influence on these values. Table 3 shows that a different number of elements was used in each animal. This is because the geometry of each individual mandible has a significant impact on mesh control. Although there were no major differences in general mandibular geometry among 
animals, the location of some anatomical landmarks differed from ewe to ewe. However, despite this uncertainty, this is not an impediment to a reliable comparative and qualitative analysis.

\section{Conclusions}

On the basis of these findings, we conclude that the FEM is capable of providing information not otherwise obtainable from clinical or experimental studies, and is highly versatile in the presence of variations in geometry, mechanical properties, and applied forces. The FEM enables calculation of the distribution of stress, force, and deformation, particularly in complex surfaces, and is an adequate substitute for in vivo determination of mechanical stresses.

It can also be concluded that neoformed bone generated by distraction osteogenesis is able to withstand and distribute masticatory forces adequately, with no critical stresses and no critical displacement of installed implants, despite a consolidation period of $<30$ days.

Finally, as a potential extension of this research project, it is suggested that a finite element analysis of the effects of variation in implant geometry, diameter, and material be conducted.

\section{Acknowledgments}

The authors thank Cláiton Heitz, Fernando V. Gomes, and Fabrício P. Massotti. This study was funded by the Brazilian National Council for Scientific and Technological Development $(\mathrm{CNPq})$.

\section{Author Disclosure Statement}

No competing financial interests exist.

\section{References}

1. Mehra, P., and Figueroa, R. (2008). Vector control in alveolar distraction osteogenesis. J. Oral Maxillofac. Surg. 66, 776-779.

2. Mofid, M.M., Manson, P.N., Robertson, B.C., Tufaro, A.P., Elias, J.J., and Vander Kolk, C.A. (2001). Craniofacial distraction osteogenesis: a review of 3278 cases. Plast. Reconstr. Surg. 108, 1103-1115.

3. Douglas, L.R., Douglass, J.B., and Smith, P.J. (2000). Intraoral mandibular distraction osteogenesis in a patient with severe micrognathia secondary to TMJ ankylosis using a tooth and bone-anchored device (PIT device): a case report. J. Oral Maxillofac. Surg. 58, 1429-1433.

4. Stewart, K.J., Weyand, B., van't Hof, R.J., et al. (1999). A quantitative analysis of the effect of insulin-like growth factor-1 infusion during mandibular distraction osteogenesis in rabbits. Br. J. Plast. Surg. 52, 343-350.

5. Freddo, A.L., Rodrigo, S.M., Massotti, F.P., Etges, A., and de Oliveira, M.G. (2009). Effect of low-level laser therapy after implantation of poly-L-lactic/polyglycolic acid in the femurs of rats. Lasers Med. Sci. 24, 721-728.

6. Takeda, Y. (1988). Irradiation effect of low-energy laser on rat submandibular salivary gland. J. Oral Pathol. 17, 91-94.

7. Fronza, B., Somacal, T., Mayer, L., de Moraes, J.F., de Oliveira, M.G., and Weber, J.B. (2012). Assessment of the systemic effects of low-level laser therapy (LLLT) on thyroid hormone function in a rabbit model. Int. J. Oral Maxillofac Surg. 42, 26-30.

8. Weber, J.B., Pinheiro, A.L., de Oliveira, M.G., Oliveira, F.A., and Ramalho, L.M. (2006). Laser therapy improves healing of bone defects submitted to autologous bone graft. Photomed. Laser Surg. 24, 38-44.

9. de Abreu, G.M., Santo, A.M., Martin, A.A., and Arisawa, E.A. (2013). Assessment of changes in mineral components in bone repair after laser therapy and pharmacotherapy by mu-EDX: a new potential tool in medical diagnostics. Photomed. Laser Surg. 31, 378-385.

10. Miloro, M., Miller, J.J., and Stoner, J.A. (2007). Low-level laser effect on mandibular distraction osteogenesis. J. Oral Maxillofac. Surg. 65, 168-176.

11. Hubler, R., Blando, E., Gaiao, L., et al. (2010). Effects of low-level laser therapy on bone formed after distraction osteogenesis. Lasers Med. Sci. 25, 213-219.

12. Cerqueira, A., Silveira, R.L., Oliveira, M.G., Sant'ana Filho, M., and Heitz, C. (2007). Bone tissue microscopic findings related to the use of diode laser $(830 \mathrm{~nm})$ in ovine mandible submitted to distraction osteogenesis. Acta Cir. Bras. 22, 92-97.

13. Zienkiewicz, O.C., and Taylor, R.L. (2002). The finite element method: Solid mechanics. Oxford: ButterworthHeinemann.

14. Hughes, T.J. (2000). The finite element method. Linear static and dynamic finite element analysis. New York: Dover Publications;

15. Geng, J.P., Tan, K.B., and Liu, G.R. (2001). Application of finite element analysis in implant dentistry: a review of the literature. J. Prosthet. Dent. 85, 585-598.

16. Akca, K., and Iplikcioglu, H. (2001). Finite element stress analysis of the influence of staggered versus straight placement of dental implants. Int. J Oral Maxillofac. Implants 16, 722-730.

17. Freddo, A.L., Hubler, R., de Castro-Beck, C.A., Heitz, C., and de Oliveira, M.G. (2012). A preliminary study of hardness and modulus of elasticity in sheep mandibles submitted to distraction osteogenesis and low-level laser therapy. Med. Oral Patol. Oral Cir. Bucal. 17, e102-e107.

18. Menicucci, G., Mossolov, A., Mozzati, M., Lorenzetti, M., and Preti, G. (2002). Tooth-implant connection: some biomechanical aspects based on finite element analyses. Clin. Oral Implants Res. 13, 334-341.

19. Han, U.A., Kim, Y., and Park, J.U. (2009). Three-dimensional finite element analysis of stress distribution and displacement of the maxilla following surgically assisted rapid maxillary expansion. J. Craniomaxillofac. Surg. 37, 145154.

20. Veziroglu, F., and Yilmaz, D. (2008). Biomechanical evaluation of the consolidation period of alveolar distraction osteogenesis with three-dimensional finite element analysis. Int. J. Oral Maxillofac. Surg. 37, 448-452.

21. Nagasao, T., Kobayashi, M., Tsuchiya, Y., Kaneko, T., and Nakajima, T. (2002). Finite element analysis of the stresses around endosseous implants in various reconstructed mandibular models. J. Craniomaxillofac. Surg. 30, 170177.

22. Duyck, J., Ronold, H.J., Van Oosterwyck, H., Naert, I., Vander Sloten, J., and Ellingsen, J.E. (2001). The influence of static and dynamic loading on marginal bone reactions around osseointegrated implants: an animal experimental study. Clin. Oral Implants Res. 12, 207-218. 
23. Ni, G.X., Choy, Y.S., Lu, W.W., et al. (2006). Nanomechanics of bone and bioactive bone cement interfaces in a load-bearing model. Biomaterials 27, 1963-1970.

24. Shimazaki, A., Inui, K., Azuma, Y., Nishimura, N., and Yamano, Y. (2000). Low-intensity pulsed ultrasound accelerates bone maturation in distraction osteogenesis in rabbits. J. Bone Joint Surg. Br. 82, 1077-1082.

25. Brunski, J.B. (1992). Biomechanical factors affecting the bone-dental implant interface. Clin. Mater. 10, 153-201.
Address reprint requests to: Marília Gerhardt de Oliveira

Rua Visconde do Herval 725 ap. 404.B, bairro Menino Deus CEP 90130-151 - Porto Alegre, RS Brazil

E-mail: gerhardtoliveira@gmail.com 